\title{
SIMMEL ET LE PROBLÈME DE LA CAUSALITÉ INDIVIDUELLE EN HISTOIRE
}

\author{
par Claude Piché, Université de Montréal
}

[ Ceci est une version de travail. Elle peut différer de la version publiée dans : Penser l'Histoire. De Karl Marx au siècle des catastrophes, C. Bouton et B. Bégout (dir.), Paris, Éditions de l'Éclat, 2011, p. 117-132. ]

Voir également ma contribution « La causalité singulière en histoire : Rickert, Simmel et Weber » déposée dans Papyrus.

RÉSUMÉ : Les problèmes de la philosophie de l'histoire de Georg Simmel s'inscrivent de plainpied dans la veine néokantienne de la « théorie de la connaissance » en s'interrogeant sur les conditions de la possibilité du discours historique. Or, afin de marquer la distance qui sépare ce discours de celui des sciences naturelles, Simmel introduit l'expression « causalité individuelle ». Par là, il maintient sans doute l'exigence scientifique d'une explication causale reposant sur une loi, mais en faisant de cette loi une « loi individuelle », il fait connaître une inflexion significative à la " nécessité » et à l' " universalité » qui se rattachent habituellement à ce concept, si bien qu'à la fin son modèle pour le discours historique devient esthétique, au sens par exemple où l'« universalité exemplaire » de l'œuvre d'art selon Kant s'avère être ici, de manière inavouée, le nouveau paradigme.

MOTS CLÉS : Simmel, histoire, causalité, loi individuelle, esthétique

ABSTARCT : Georg Simmel's Problems of the Philosophy of History subscribe to the neoKantian 'epistemological' approach in the sense that it raises the question of the conditions of the possibility of the historical discourse. In order to stress the gap that prevails between this discourse and the natural sciences, Simmel coins for the historical sciences the expression "individual causality". To be sure, he maintains thereby the scientific requirement of a causal explanation based on a law, but by making this law an "individual law," he transforms the usual meaning of the "necessity" and the "universality" usually attached to this concept, so much so that in the end his conception of the historical discourse becomes aesthetic. This means that the Kantian "exemplary universality" of the work of art becomes here, implicitly, the new paradigm.

KEY WORDS : Simmel, history, causality, individual law, aesthetics 
L'entendement tient tellement aux règles qu'il se trouve souvent repoussé dans ses derniers retranchements. Et qu'on enlève une seule fois les règles, et on ne sait plus alors à quoi s'en tenir.

Kant, Abrégé de philosophie.

Dans son texte intitulé Comprébension et politique (1953), Hannah Arendt formule les remarques suivantes à propos de la causalité en histoire :

À vrai dire, dans le domaine des sciences historiques, la causalité n'est qu'une catégorie totalement déplacée et source de distorsion... Il ne saurait survenir d'événements, c'est-àdire de phénomènes d'une irréductible nouveauté, dans ce cadre de catégories préconçues, dont la plus frustre est la notion de causalité : une histoire dépourvue d'événements fait place à la monotonie sans vie du même, déployé dans le temps, l'eadem sunt omnia semper de Lucrèce

1 .

Le message est on ne peut plus clair. La causalité est désignée ici comme cette catégorie qui n'a pas sa place en histoire, sauf à vouloir se résoudre à n'y trouver que la monotonie de ce qui se répète constamment. Or, là n'est précisément pas le but de l'histoire. Au contraire, ce qui aux yeux d'Arendt présente un intérêt, c'est l'irruption du singulier, de l'unique, bref de la nouveauté. Le recours au concept de causalité soulève dès lors un problème particulier qui ressortit à la méthodologie de l'histoire.

Au début du XXe siècle, la question de la méthode des sciences historiques a retenu l'attention, comme on le sait, d'un certain nombre de philosophes néo-kantiens en Allemagne. N'entendant nullement renouer avec la philosophie de l'histoire au sens traditionnel, ils ont plutôt abordé cette discipline par le biais d'une épistémologie ou encore d'une théorie de la connaissance. Inspirés par Kant, ils n'hésitèrent pas à traiter la question de manière radicale, en la situant au plan transcendantal. Dans ce qui suit, j'aimerais attirer l'attention tout particulièrement sur le travail de Georg Simmel qui, avec Heinrich Rickert, fut l'un des nombreux interlocuteurs de Max Weber, notamment au moment où ce dernier s'appliquait à développer une méthodologie « compréhensive » pour les sciences historiques et sociales. Je pense entre autres à l'ouvrage de Simmel intitulé Les problèmes de la philosophie de l'histoire, paru d'abord en 1892, puis coup sur coup dans une seconde et une troisième édition en 1905 et 1907. Dans la préface à la deuxième édition, Simmel déclare sans ambages son intention : renouveler à l'endroit des disciplines historiques le geste posé par Kant pour la science de la nature en dégageant les « catégories » qui sont propres à cet objet qu'est l'histoire. Il ne faut donc pas s'étonner de voir Simmel formuler son projet sous la forme d'une question, d'allure éminemment kantienne : «Comment l'histoire est-elle possible ? ». Cette question mérite en fait

\footnotetext{
${ }^{1}$ Hannah Arendt, « Compréhension et politique », dans La nature du totalitarisme, tr. M.-I. B. de Launay, Paris, Payot, 1990,
} p. 54 et 56 . 
d'être posée de manière autonome car le problème qu'elle soulève ne peut être simplement subsumé sous l’interrogation originale de Kant : «Comment la nature est-elle possible ?»

D’après Simmel, en effet, la présentation que fait Kant des catégories de la connaissance est en vérité taillée sur mesure pour le discours des sciences naturelles, que ces concepts ont pour but de fonder. Ce sont les catégories comme concepts purs de l'entendement qui sont à l'origine de la prétention à l'objectivité de ces disciplines. On connaît en outre la place centrale chez Kant de la catégorie de causalité et du principe auquel elle donne lieu. C’est ce principe qui autorise la formulation de lois régissant des enchaînements de phénomènes d'une régularité sans faille. Parce que le principe philosophique de causalité est un principe a priori, il fonde la prétention à la nécessité et à l'universalité des lois de la physique mathématique. Plus précisément, ce principe a priori établit 1) la nécessité du lien entre la cause et l'effet et 2) l'universalité de cette liaison qui stipule que l'effet suit invariablement la cause en vertu d'une « règle ». Et c'est ce dernier trait qui pose problème pour les disciplines historiques. Le principe kantien de causalité précise en effet qu'il n'y a causalité que là où il y a une règle générale. Ainsi, parce que le principe consacre le caractère répétitif des séquences causales et leur prévisibilité, il semble plutôt mal convenir à la science historique, qui comme ne vise pas à établir des lois.

Simmel se doit donc de marquer une certaine distance vis-à-vis de Kant. Il estime que lorsque la théorie de la connaissance aborde l'objet histoire, elle doit envisager de nouveaux a priori, propres à celle-ci, et renouveler l'usage des catégories. Car exiger de la part de l'histoire d'établir des lois constantes du devenir équivaudrait tout bonnement à lui demander de détruire son objet spécifique. Tel est le constat auquel il parvient à l'issue de l'examen de cette question : « Ainsi l'exigence de soumettre à des lois le devenir que l'on désigne comme histoire dissout à la fin le concept spécifique d'histoire »². L’un des principaux enjeux de l'épistémologie de Simmel consiste donc à surmonter cette opposition entre l'individualité historique, d'une part, et le nécessaire et l'universel, de l'autre.

Il convient d'entrée de jeu de le préciser, Les problèmes de la philosophie de l'histoire de Simmel contiennent tout autre chose qu'une simple tentative pour résoudre ce problème. Nous faisons face ici à un texte d'une redoutable complexité, que l'on sent animé par la préoccupation de serrer au plus

\footnotetext{
${ }^{1}$ Kant, Critique de la raison pure, A 189, tr. A. J.-L. Delamarre et F. Marty, dans le même CEuvres philosophiques I, Paris, Gallimard, Biblioth. de la Pléiade, 1985, p. 925.

${ }^{2}$ Georg Simmel, Die Probleme der Geschichtsphilosophie. Eine erkenntnistheoretische Studie (1905/1907), 2e et 3e éd., dans le même, Gesamtausgabe, Tome 9, Francfort-sur-le-Main, Suhrkamp, 1997, p. 337; tr. R. Boudon, Les problèmes de la philosophie de l'histoire. Une étude d'épistémologie, Paris, PUF, 1984. p. 160. Toutes les traductions des textes de Simmel sont les miennes.
} 
près l'objet histoire dans toute sa richesse et dans ses multiples strates. C'est Raymond Boudon qui affirme de cet ouvrage qu'il est tout simplement impossible à résumer ${ }^{1}$. Il contient un foisonnement d'idées développées de manière subtile et judicieuse, idées dont cependant on ne perçoit pas toujours l'unité systématique. Pour ma part, je ne retiendrai que deux éléments parmi les nombreux thèmes traités, et de surcrô̂t il s'agit de deux éléments que Simmel considère lui-même comme des hypothèses : 1) la causalité individuelle et 2) l'analogie entre le discours de l'historien et l'œuvre d'art. Ainsi, le premier élément est-il qualifié de «supposition audacieuse »² et risquée. D’ailleurs, les commentateurs ont tôt fait de noter que l'expression causalité individuelle a toutes les apparences d'une contradiction dans les termes ${ }^{3}$. Quant à l'analogie avec l'art, elle ne représente pour Simmel qu'une « première tentative », qu'une simple « suggestion » ${ }^{4}$ en vue de résoudre le problème soulevé. Sans doute, ces deux thèmes occupent-ils une place somme toute limitée dans l'ouvrage mais, à titre d'hypothèses de travail, ils procèdent d'une volonté très nette de cerner la spécificité de l'histoire et du discours qui lui est consacré.

1 - Causalité individuelle : le maintien du concept de loi.

Les quelques pages consacrées à la causalité individuelle dans le second chapitre de l'ouvrage prennent pratiquement l'allure d'un hors d'œuvre puisqu'elles sont rassemblées sous la forme d'une simple « remarque ». L'expression causalité individuelle parle en fait d'elle-même. Elle vise à déstabiliser le lecteur qui à l'habitude de voir la causalité associée sans plus au concept de loi, d'une loi universelle, valable dans tous les cas. Ce qui signifie non seulement que la cause A entraîne nécessairement l'effet $\mathrm{B}$, mais qu'il en va ainsi à chaque fois que $\mathrm{A}$ se présente. Le lien entre $\mathrm{A}$ et $\mathrm{B}$ est bien sûr nécessaire, mais cette nécessité s’exprime par le caractère pour ainsi dire « légal » du rapport.

Simmel convie alors son lecteur à une expérience de pensée. Il lui demande de sous-peser une simple possibilité logique afin de voir si l'on peut se dégager du dogme kantien de la loi causale classique. Il nous invite donc à considérer l'hypothèse suivante : si, dans le monde actuel, A entraîne toujours B, mais que dans un autre monde possible ce même A pouvait en revanche entraîner C,

\footnotetext{
${ }^{1}$ Raymond Boudon, «Introduction » à sa traduction de Les problèmes de la philosophie de l'histoire, p. 51.

2 Simmel, Die Probleme der Geschichtsphilosophie (1905/1907), p. 314.

3 Voir à ce sujet H.-J. Dahme et O. Rammstedt, «Einleitung » à G. Simmel, Schriften zur Soziologie, Francfort-sur-le-Main, Suhrkamp, 1983, p. 18.

4 Simmel, Die Probleme der Geschichtsphilosophie (1905/1907), p. 275.
} 
pourquoi ne serions-nous pas autorisés à imaginer que dans le monde actuel A puisse, à un certain moment, causer B et, à un autre moment, entraîner C. Il n'y a là aucune contradiction. En d'autres mots, on nous enjoint d'envisager la possibilité pour un enchaînement causal de n'avoir qu'une seule et unique occurrence. Dans ces conditions, une même cause pourrait avoir des effets variables dans le temps et donner lieu à des séquences d'événements différents. Un tel schéma semble requis, selon Simmel, pour l'examen de la dynamique interne à la vie psychique ${ }^{1}$. La causalité individuelle permet en effet de faire une place à la productivité de la vie intérieure, à sa créativité et aux multiples liaisons de représentations que l'on y trouve. L'enjeu consiste donc à neutraliser la violence qui est traditionnellement faite au singulier par le caractère générique du concept et de mettre un terme à la répression dont fait l'objet, pour reprendre l'expression d'Adorno, le «non-identique ». Du reste, on ne s'étonnera pas d'apprendre que ce dernier a consacré une conférence au thème spécifique de la causalité individuelle chez Simmel, conférence prononcée en 1940 à l’Université Columbia ${ }^{2}$.

Or le plus étonnant dans l'hypothèse de Simmel, c'est qu'il renonce, contrairement à Rickert ${ }^{3}$, à rejeter le concept de loi lorsqu'il prend congé de la régularité et de l'itérativité de l'enchaînement causal en histoire. Il faut lire attentivement les énoncés consacrés à ce sujet dans son ouvrage. Ainsi à propos du caractère répétitif de la séquence $\mathrm{A}-\mathrm{B}$ dans les sciences de la nature, il prend soin de joindre un qualificatif à la loi qui régit une telle séquence : «Or le lien entre la causalité et une loi de ce genre ne m'apparaît pas logiquement indispensable ${ }^{4}$. Sa critique porte en fait uniquement sur une loi « de ce genre », laissant entendre que toute loi n’implique pas d'entrée de jeu une telle prétention à la réitérabilité. Évidemment, ce qu’il propose ici a quelque chose de tout à fait inédit : s'il existe quelque chose de tel qu'une loi de la causalité individuelle, celle-ci s'avère être une loi « unique en son genre » (einzigartige Gesetəlichkeeit ${ }^{5}$ ), il s'agit de la loi qui appartient en propre à une individualité ${ }^{6}$. Il convient dès lors de préciser que cette loi a ceci de particulier qu'elle ne rend pas prévisible le comportement de l'individu. Elle correspond à l'expérience de pensée déjà évoquée : il y aurait une variabilité constitutive de cette causalité et de sa loi, si bien que cette dernière ne pourrait permettre d'anticiper le comportement de l'individu historique. À un certain moment, A causerait B,

\footnotetext{
1 Simmel, Die Probleme der Geschichtsphilosophie (1905/1907), p. 315.

2 Theodor W. Adorno, «Über das Problem der individuellen Kausalität bei Simmel », dans Frankfurter Adorno Blätter VIII, R. Tiedemann (dir.), edition text + kritik, 2003, p. 42-59.

${ }^{3}$ Heinrich Rickert, Der Gegenstand der Erkenntnis, Tübingen, J. C. B. Mohr, 1904, p. 212, 224; Die Probleme der Geschichtsphilosophie. Eine Einführung (1904), Heidelberg, Carl Winter Universitätsverlag, 1924, p. 48-49; et Wilhelm Windelband, Tübingen, J. C. B. Mohr, 1929 (2e éd.), p. 21.

4 Simmel, Die Probleme der Geschichtsphilosophie (1905/1907), p. 313.

5 Simmel, Die Probleme der Geschichtsphilosophie (1905/1907), p. 316.

6 Simmel, Die Probleme der Geschichtsphilosophie (1905/1907), p. 317.
} 
et à un autre, A entraînerait $C$ etc. Sa loi n'engendrerait donc pas ici de schémas d'action que l'on pourrait qualifier de typiques de tel ou tel individu.

À l'évidence, Simmel est fort conscient des limites de son modèle théorique. Philosophe averti, il sait que nous ne disposons d'aucun moyen pour identifier cette loi et pour lui attribuer avec certitude quelque effet que ce soit. L'absence de régularité dans l'enchaînement des événements psychiques fait en sorte que cette loi de l'individu est en vérité impossible à déceler. Elle échappe à l'emprise de la connaissance, si bien que sous ce rapport l'enchaînement causal singulier ne peut être distingué d'une simple succession fortuite d'événements. Puisque le cours des événements régi par cette loi à l'intérieur de l'individu ne laisse rien anticiper du comportement à venir, comment dès lors faire le départ entre un comportement ainsi régi et le pur hasard?

Face à l'événement unique et incomparable quant à son contenu, nous ne disposons d'aucun moyen pour distinguer les divers moments de l'authentique causalité dont il est question ici, de la simple succession dans le temps, contingente et dépourvue de liens intrinsèques ${ }^{1}$.

La difficulté théorique que pose la causalité individuelle est de taille, en dépit de l'intérêt que présente ce modèle. C'est que la loi qui règne au sein de l'individu, parce qu'elle ne peut être connue, demeure parfaitement opaque pour l'observateur extérieur, donc pour l'historien. Rien dans ses occurrences ponctuelles ne permet selon Simmel de la distinguer du hasard aveugle ou encore, pourrait-on ajouter, de la nécessité aveugle. Ce sont là deux expressions dont Kant fait usage dans la Critique de la raison pure lorsqu'il traite des principes de la modalité ${ }^{2}$. À ses yeux, il est impossible de distinguer dans l'expérience ce qui est purement fortuit de ce qui est en soi-même nécessaire, puisque dans les deux cas la succession temporelle ne repose sur aucun lien dynamique. Celle-ci ne contient pas d'enchaînement proprement dit, mais une simple succession, qu'il faut comprendre comme une juxtaposition d'événements. Or, Kant montre que la nécessité pour le sujet connaissant fini ne peut être saisie que si elle est hypothétique. En d'autres mots, l'occurrence d'un événement doit être comprise comme l'effet d'une cause qui précède dans le temps ${ }^{3}$. Le schéma de la nécessité

\footnotetext{
${ }^{1}$ Simmel, Die Probleme der Geschichtsphilosophie (1905/1907), p. 314.

${ }^{2}$ Kant, Critique de la raison pure, A 227-228/B 280 ; tr., p. 960.

3 Voir aussi Kant, Prolégomènes, $\int$ 60: « ...toute nécessité naturelle dans le monde sensible est toujours conditionnée, en tant qu'elle suppose constamment la dépendance de toute chose à l'égard des autres...», CEuvres philosophiques II, p. 149. Le caractère «conditionné » de la nécessité marque la dépendance d’un événement en regard de la cause qui le précède, alors que la nécessité aveugle exprime une nécessité sans attaches dans le monde réel. "Kant entend par Fatum ce qui arriverait en vertu d'une nécessité aveugle, par laquelle certains événements seraient fixés en eux-mêmes, indépendamment des causes qui les produisent. » André Lalande, Vocabulaire technique et critique de la philosophie, Paris, PUF, 1976, article « Fatum », p. 345-346. Il s'agit là précisément de la fatalité que Leibniz qualifie de fatum mahometanum: "Je viens de montrer comment l'action de la volonté dépend de ses causes, qu'il n’y a rien de si convenable à la nature humaine que
} 
hypothétique fait donc forcément référence à une condition et il s'exprime dans la formule : si... alors... Or ce schéma chez Kant doit répondre à une exigence d'universalité, comme nous l'avons $\mathrm{vu}$ : à toutes les fois que la condition se présente, le conditionné s'ensuit, et ce en vertu d'une règle ${ }^{1}$. Et c'est précisément cette conception de l'universalité, entendue comme valable dans tous les cas, que tente de contrer Simmel dans son épistémologie de l'histoire. Le tribut à payer pour cette exclusion de la loi « universelle » est toutefois très lourd, car on se prive de l'avantage souligné par Kant : en vertu de la causalité à l'œuvre dans le champ des sciences naturelles, la nécessité de l'effet devient « intelligible » du fait d'être conditionnée conformément à une règle constante. Si l'on s'entend pour compter Simmel au nombre des théoriciens de l'approche « compréhensive » en histoire, force nous est de constater qu'il se prive sciemment de la dimension de «Verständlichkeit» évoquée par Kant à propos de la causalité physique. La composante de nécessité que Simmel tient malgré tout à conserver grâce à la loi « unique en son genre » et qui lui permet d'éliminer le pur arbitraire dans le cours des phénomènes psychiques, ne peut en vérité être identifiée. Qu'à cela ne tienne. Il y a tout lieu de croire, selon Simmel, que ce modèle cerne l'objet historique de beaucoup plus près que ne le fait le principe kantien de causalité, tourné exclusivement vers les phénomènes naturels dans leur redondance. À tout le moins ce modèle mérite-t-il à ses yeux d'être pris sérieusement en considération (erwähnenswert²).

Max Weber, dans ses écrits méthodologiques, se voit pour sa part incapable de faire place à une causalité individuelle ainsi conçue. Il soutient que l'intelligibilité en histoire requiert de façon impérieuse le recours à ce qu'il est convenu d'appeler les possibilités objectives et la causalité adéquate $^{3}$. Sans l'apport de l'explication causale, il est à vrai dire très difficile d'accéder à l'intelligence des phénomènes historiques. Selon lui, la compréhension méthodiquement contrôlée de ces phénomènes n'est pas envisageable sans référence à une causalité qui se répète. À l'évidence, plusieurs genres de règles peuvent entrer en jeu ici, pour autant toutefois qu'elles permettent de décrypter les comportements. Car, en dernière analyse, la compréhension du phénomène historique trouve son point d'appui dans l'explication causale. « [La] compréhension seule n'est jamais valable

cette dépendance de nos actions, et qu'autrement on tomberait dans une fatalité absurde et insupportable, c'est-à-dire dans le fatum mahometanum, qui est le pire de tous, parce qu'il renverse la prévoyance et le bon conseil. Essais de théodicée, Paris, Garnier-Flammarion, 1969, p 136, \59.

${ }^{1}$ Kant, Critique de la raison pure, A 198/B 243-44, tr., p. 933.

${ }^{2}$ Simmel, Die Probleme der Geschichtsphilosophie (1905/1907), p. 318.

3 Max Weber, « Roscher und Knies und die logischen Probleme der historischen Nationalökonomie » (1903-1906), dans le même Gesammelte Aufsätz̧e zur Wissenschaftslehre, J. Winkelmann (dir.), Tübingen, J.C.B. Mohr, 1973, p. 69. Cf. Ernst Troeltsch, Der Historismus und seine Probleme (1922), Aalen, Scientia, 1961, p. 566; de même que mon article « De Max Weber à Heidegger. L'historiographie comme liquidation méthodique de l'histoire ", Critique 461, 1985, p. 955-973. 
objectivement », précise Raymond Aron à propos de Weber, « elle doit être vérifiée par la causalité » Cette causalité comprise dans un sens très large fait donc appel à toutes les ressources à la disposition de l'historien, qu'il s'agisse des lois dégagées par la psychologie ou du bagage acquis par le chercheur au hasard de ses expériences de vie (Lebenserfahrungen² ${ }^{2}$. Bref, tout ce que Weber désigne par l'expression « savoir nomologique ».

Comme Simmel ne cite que très rarement ses contemporains épistémologues, il nous faut établir nous-mêmes la confrontation, si sommaire soit-elle, entre les deux positions. En fait, ces deux auteurs partagent plusieurs éléments en vue de la mise sur pied d'une méthode compréhensive pour les sciences humaines. L'ouvrage de Simmel regorge d'indices qui montrent à quel point le savoir nomologique wébérien entre en ligne de compte pour l'interprétation des phénomènes historiques. Les deux auteurs y ont toutefois recours dans une perspective radicalement opposée. Si, pour Weber, la compréhension repose ultimement sur l'intelligibilité issue d'un schéma d'explication causale fondée sur des règles, chez Simmel, en revanche, la compréhension de l'individualité historique ne doit surtout pas se limiter aux ressources que procure cette forme d'intelligibilité. À vrai dire, ce dernier considère la connaissance acquise au fil des expériences ainsi que les régularités psychologiques comme une simple « étape préalable » (Vorstufe $e^{3}$ à une véritable compréhension de la personnalité historique dans son unicité. Évidemment, les concepts généraux, le savoir nomologique et les diverses typologies ${ }^{4}$ peuvent fournir une première approche de la particularité de l'individu, mais ils le font en dégageant des traits que ce dernier a tout de même en partage avec d'autres. Or, la visée ultime de Simmel consiste à exposer l'individualité dans ce qu'elle a de plus singulier, dans ce que la personnalité historique a d'unique. D'où l'importance de marquer ses distances vis-à-vis des concepts génériques et des lois générales.

En ce sens, la loi propre à l'individu, celle qui n'est pas formulable conceptuellement mais qui imprègne une nécessité à sa biographie, était une première tentative pour rendre justice à cette unicité, tentative du reste sur laquelle Simmel reviendra quelques années plus tard, au plan de l'éthique cette fois, avec le thème de la « loi individuelle » ${ }^{5}$. Bien sûr, par le texte de 1913 portant ce titre, Simmel quitte le registre de l'épistémologie de l'histoire pour passer à celui de la morale. Mais

\footnotetext{
${ }^{1}$ Raymond Aron, La philosophie critique de l'histoire. Essai sur une théorie allemande de l'bistoire, Paris, Vrin, Coll. « Points », 1969 , p. 241.

2 Weber, «Die Objektivität sozialwissenschaftlicher und sozialpolitischer Erkenntnis » (1904), Gesammelte Aufsätze zur Wissenschaftslehre, p. 179.

${ }^{3}$ Simmel, Die Probleme der Geschichtsphilosophie (1905/1907), p. 268.

4 Simmel, «Bruchstücke aus einer Philosophie der Kunst » (1916), dans le même, Das Individuelle Gesetz. Philosophische Exkurse, M. Landmann (dir.), Francfort-sur-le-Main, Suhrkamp, 1987, p. 111.

${ }^{5}$ Simmel, « Das individuelle Gesetz » (1913), dans Das individuelle Geset», p. 174-230.
} 
on perçoit sans difficulté que l'expression « loi individuelle » fait écho, à sa manière, au problème de la causalité individuelle que nous venons d'examiner. C'est dire que l'hypothèse avancée à propos de l'histoire était plus qu'une simple illumination passagère de la part de Simmel. L'enjeu en éthique est en effet similaire et c'est encore une fois l'étroitesse de la vision kantienne qui est mise au banc des accusés. L'essai intitulé La loi individuelle peut en fait être interprété comme une polémique menée de manière systématique contre la conception kantienne de la loi morale. Le devoir contenu dans l'impératif catégorique commande l'universalisation de toute maxime, en sorte qu'une telle morale fait violence au caractère pour ainsi dire protéiforme de la vie morale en lui imposant le carcan de l'uniformité. En ce sens, la loi morale kantienne reproduit la même exigence d'universalité que la loi naturelle ou encore, comme l'avait déjà montré Schleiermacher, elle entraîne le même nivellement que la loi juridique ${ }^{1}$ dont Kant s'est malencontreusement inspiré pour sa morale. En vertu de cette dernière forme de législation, il va de soi que tous sont égaux devant la loi, tout comme en vertu de la loi générale de la nature, les phénomènes sont appelés à se répéter sans cesse. À l'instar de Hannah Arendt, Simmel se montre soucieux de conjurer le spectre de l'« éternel retour $»^{2}$ dans les affaires humaines.

2 - Le paradigme esthétique. La signification universelle de l’individualité historique.

Nous avons vu que ce n'est qu'avec difficulté que Simmel parvient à relever le défi qu'il s'est lancé dans son ouvrage : réconcilier l'individualité la plus stricte avec le nécessaire et l'universel. Or, c'est ici qu'intervient le paradigme esthétique, développé aux Chapitres I et III de l'ouvrage. Il importe de préciser que cette dimension concerne l'historien lui-même, dans la mesure où c'est lui que est responsable de la mise en récit de la trame historique. Et d'emblée Simmel s'empresse de faire une mise en garde. L'aspect esthétique de la narration des événements historiques ne correspond pas à la composante simplement rhétorique du récit. Loin d'avoir uniquement une fonction cosmétique et par là même accessoire en histoire, le paradigme esthétique est appelé à jouer un rôle tout à fait central. Car il faut préciser que l'approche néo-kantienne défendue ici s'oppose de manière frontale au réalisme historique. Ce qui signifie que l'objet histoire prend forme grâce à l'intervention de l'historien. C'est en réalité l'historien qui fait l'histoire, qui donne sens au devenir

\footnotetext{
1 Simmel, «Das individuelle Gesetz », p. 182-183.
}

2 Simmel, « Das Individuelle Gesetz », p. 190. 
historique, ou plus précisément au parcours de l'individualité historique. Voyons d'un peu plus près ce qu'il en est de cette question de l'accès à l'objet.

C’est Dilthey qui a insisté à juste titre sur le fait que dans le champ des sciences humaines, l'esprit rencontre dans son objet thématique un autre esprit. Or, cet objet thématique n'est sans doute pas aussi malléable que les corps inanimés du physicien. L’objet de l'historien est donc en partie formé, préformé pour ainsi dire. Toutefois, notre examen de la causalité individuelle a révélé que pour ce qui intéresse le plus l'historien, c'est-à-dire la personnalité historique dans son unicité, celle-ci demeure réfractaire à une analyse causale standard. Par ailleurs, la causalité individuelle imputée à l'agent historique ne donne, en dernière analyse, rien à comprendre de ce qui fait l'essentiel d'une individualité. C'est pourquoi le discours historique se voit assigner la tâche de procéder à une reconstruction du fil des événements pour en faire l'objet d'une narration, c'est-à-dire une totalité douée de sens. Le terme employé par Simmel ici, «reconstruction », traduit très précisément son intention : les éléments rattachés à une individualité historique constituent d'entrée de jeu des matériaux épars qu'il s'agit de mettre en forme, ce qui implique une intervention active de l'historien, essentielle à son travail.

L'analogie qu'entend faire Simmel entre le travail de l'artiste et l'œuvre de l'historien se situe donc au niveau le plus originaire de l'historiographie. Il insiste beaucoup sur ce parallèle, bien qu'il sache par ailleurs fort bien que des distinctions importantes doivent être faites. En effet, l'œuvre d'art se distingue de l'objet historique en ceci qu'elle est coupée de la réalité dynamique du monde réel. Elle est sous ce rapport « autosuffisante » puisque dégagée par définition des multiples chaînes causales qui s'entrecroisent dans la réalité du monde. Or, bien qu'en revanche l'objet historique soit partie intégrante de ce monde concret, la tâche de l'historien s'apparente en ceci à celle de l'artiste que de part et d'autre l'on s'emploie à constituer une totalité significative. Si c'est véritablement une méthodologie compréhensive que vise Simmel pour l'histoire, il incombe à l'historien de montrer le sens des événements. Mais comment peut-il le faire tout en respectant les canons de la scientificité : à savoir l'exigence d'universalité et de nécessité? Contre toute attente, c'est précisément à ce chapitre que l'analogie avec le modèle de l'œuvre d'art s'avère la plus féconde aux yeux de Simmel.

Si l'on peut prétendre que l'œuvre d'art accède, à sa manière, à l'universalité, il nous faut d'emblée définir ce terme, auquel nous nous attarderons en premier lieu pour ensuite passer au problème de la nécessité. En art, l’universalité recherchée ne concerne pas l’application d'un

\footnotetext{
${ }_{1}$ Simmel, «Das individuelle Gesetz », p. 206. Voir du même auteur Kant. Sechžehn Vorlesungen gehalten an der Berliner Universität (1904), Gesamtausgabe, Tome 9, p. 202.
} 
concept, mais plutôt ce qu'il nous faut bien appeler, faute d'une meilleure expression, l'universalité de l'adhésion. L'œuvre est en vérité à la recherche de l'unanimité de la part des spectateurs, dans la mesure où ceux-ci sont spécifiquement appelés à en reconnaître la portée. L’unanimité en question, celle qui est recherchée, est donc le consensus de tous les spectateurs en face d'une œuvre qui demeure pourtant unique. Transposée à la science historique, cette recherche d'unanimité de la part de l'historien concerne sa reconstruction d'une personnalité unique.

Tant en art qu'en histoire, on constate que l'unanimité touche ce qui est valable pour tous, sans pour autant que cela soit commun à tous à la manière, par exemple, dont divers cas tombent sous un concept général.

Dès lors, ce qui est tout à fait individuel, en dépit du fait qu'il ne se soit produit qu'une seule fois dans l'histoire, recèle pourtant une essence humaine universelle pour ainsi dire, qui est d'une certaine manière plus intemporelle que les représentants existant en plusieurs exemplaires d'une situation spatio-temporelle déterminée ${ }^{1}$.

Tout comme la validité universelle à laquelle prétend l'œuvre d'art, la validité de la reconstruction historique tient à sa signification pour l'humanité, à un « sens » qui lui confère une portée intemporelle. Pour reprendre les termes de Simmel, il ne s'agit pas ici d'une universalité qui produit l'unification « de plusieurs choses » sous un concept général, mais d'une universalité qui, grâce au travail de l'historien, produit l'unanimité d'une «multitude... de sujets » (Seelen²). L'historien détient en fait le secret de mettre en forme la signification d'une constellation historique à laquelle tout un chacun est appelé à souscrire. Chacun est invité à reconnaître ce sens, à se reconnaître dans l'objet narré et à y prendre part.

Conférer ce genre d'universalité à l'individuel, c'est-à-dire à ce qui est tout à fait unique, voilà le secret artistique de l'historien, dans lequel se révèle de manière on ne peut plus claire ce qui dans sa science ne peut être appris ${ }^{3}$.

On en conviendra, cette analogie avec le travail de l'artiste conduit aux limites du discours rationnel. Le savoir-faire de l'historien parvient à porter au jour la validité universelle d'une individualité historique dans la mesure même où ce savoir-faire transcende la discursivité et sa logique. Le métier d'historien sous ce rapport ne peut être appris. Il ne peut faire l'objet d'un savoir transmissible. Dans ces conditions, il convient de se demander si une telle méthodologie peut encore faire droit à l'exigence de nécessité.

\footnotetext{
1 Simmel, Die Probleme der Geschichtsphilosophie (1905/1907), p. 304-305.

2 Simmel, Die Probleme der Geschichtsphilosophie (1905/1907), p. 298.

3 Simmel, Die Probleme der Geschichtsphilosophie (1905/1907), p. 298.
} 
Nous avons vu que du point de vue de l'explication causale, le discours historique ne peut s'en remettre à des lois psychologiques ou encore à un quelconque savoir nomologique sous peine de dissoudre, à terme, la singularité constitutive de l'objet historique. Bien que Simmel ait cherché à maintenir le caractère nécessaire des liens entre les éléments psychiques de l'acteur historique, la loi singulière et insondable sur laquelle est censée reposer cette nécessité interdit tout jugement d'imputation causale intelligible. Au plan de la causalité psychologique individuelle, la nécessité était donc condamnée à demeurer une simple supposition de la part de Simmel. Mais cette difficulté ne doit pas représenter un obstacle insurmontable pour l'historien. À l'évidence, son matériau de départ est constitué pour une large part des vécus de l'agent historique, mais le discours historique, précisément en ce qu'il présente d'analogue à l'œuvre d'art, dispose d'autres ressources. L'historien n'est nullement contraint de s'en tenir aux matériaux psychiques dans l'ordre, voire dans le désordre au sein duquel ils se présentent à lui. Si les liens dynamiques entre les phénomènes psychiques demeurent obscurs au niveau de l'agent historique, rien ne s'oppose à ce que l'historien réorganise ces matériaux pour ses propres fins. Mieux : il se doit d'entreprendre une telle mise en perspective des événements, psychiques ou autres. À l'instar de l'artiste, il agence ces matériaux à nouveaux frais et produit un ensemble obéissant à une logique propre et visant à mettre en évidence la signification universelle de l'objet à l'étude, signification qui n'est en rien tributaire d'une analyse des causes efficientes.

Dès lors, puisque les lois générales ne se situent pas au cœur d'une telle reconstruction, la nécessité doit se manifester d'une autre manière. Elle est en fait attestée par le même indice que l'on trouve dans l'appréciation de l'œuvre d'art : le sentiment. C'est dire que dans son épistémologie des sciences historiques, Simmel n'a aucune réticence à recourir à une nécessité qui trouve son siège dans la subjectivité de l'historien et de ses lecteurs. Le caractère non arbitraire des éléments qui sont incorporés dans la présentation est ainsi attesté par le sentiment. Simmel peut alors dire de l'historien qu'il

réalise cette construction psychologique avec un sentiment de nécessité et [qu'] il la distingue très exactement de toute autre liaison de représentations, bien que cette construction ne puisse avoir lieu à cet instant qu'en lui et n’être légitimée par aucune loi concrète... 1 .

C'est donc de cette manière que Simmel entend gagner son pari. Le discours historique est porteur de nécessité tout autant qu'il élève une prétention à l'universalité. Si la causalité singulière qui préside à la liaison nécessaire des représentations se soustrayait au regard de l'observateur, voilà qu'en

\footnotetext{
1 Simmel, Die Probleme der Geschichtsphilosophie (1905/1907), p. 268.
} 
revanche l'art de l'historien instaure une nécessité d'une autre nature, cette fois au plan de la composition de son discours, c'est-à-dire au plan de la constellation des événements qu'il met en scène. Il satisfait par là, du moins dans un sens bien particulier, aux réquisits d'universalité et de nécessité tout en évitant de tomber dans le piège qui consiste à faire de l'histoire une discipline nomothétique.

La construction d'ensembles psychiques qui est accompagnée du sentiment immédiat de cohérence et qui par là même constitue la seule possibilité de comprendre l'événement historique dont le sujet est porteur, cette construction donc implique une synthèse tout à fait unique de la catégorie de l'universel et du nécessaire d'une part, et de celle de l'individuel pur de l'autre...

Il convient de noter que le mot « comprendre » est souligné par Simmel dans cet extrait. L'individualité accède par là à une certaine forme d'intelligibilité qui justifie la pertinence des catégories d'universalité et de nécessité pour le discours historique. Le sentiment de nécessité ressenti par le lecteur du récit cautionne la validité universelle de la constellation historique esquissée par l'historien. Une forme de compréhension est donc maintenue, qui court-circuite la voie logique qu'empruntent habituellement les sciences pour garantir l'objectivité de leurs résultats. Il faut bien prendre la mesure de ce que l'on nous propose ici, c'est-à-dire de l'audace de Simmel. Le discours historique possède à n'en pas douter un critère, mais l'universalité de celui-ci demeure malgré tout subjective. Peut-on prétendre dès lors que ce discours accède à la vérité ? Simmel n’en disconviendrait sans doute pas. Mais c'est un fait qu'il n'utilise que rarement ce mot pour désigner le but visé par les sciences historiques. Et lorsqu'il le fait, il se sert, par exemple, de l'expression Lebenswabrheit'. En fait, le modèle esthétique qu'il transpose à l'histoire entraîne un certain nombre de conséquences. Comme l'œuvre d'art, le discours historique aspire à la Richtigkeit ${ }^{3}$, ce critère non conceptualisable, ce je-ne-sais-quoi, qui permet de distinguer l'œuvre aboutie de l'œuvre ratée ${ }^{4}$.

\footnotetext{
1 Simmel, Die Probleme der Geschichtsphilosophie (1905/1907), p. 269. Cf. « Das individuelle Gesetz », p. 220.

2 Simmel, Die Probleme der Geschichtsphilosophie (1905/1907), p. 269.

${ }^{3}$ Simmel, Die Probleme der Geschichtsphilosophie (1905/1907), p. 275, cf 267, 356.

${ }^{4}$ Lorsque Heinrich Rickert dans son article «Les quatre modes de l'universel en histoire » présente le type d'universalité que nous venons d'exposer, il s'abstient bien d'établir quelque rapprochement que ce soit entre ce second mode de l'universel en histoire et l'art. En fait, il renonce à toute allusion au paradigme esthétique, sans doute parce qu'il se définit d'abord comme logicien et qu'il tient à prendre ses distances vis-à-vis de son collègue Windelband qui avait initialement associé l'approche des sciences historiques individualisantes à celle de l'artiste en insistant sur les « formes » (Gestalten) que produit l'historien, par opposition aux lois que s'applique à établir le physicien. C'est sans doute par crainte de se voir associé au romantisme que Rickert maintient une définition simplement formelle de cet universel qui vaut pour tous dans la mesure précise où tous ne l'ont pas en partage (du moins pas au même degré que le modèle incarné par l'individualité historique). L'article de Rickert est paru dans la Revue de synthèse historique 2, 1901, p. 128-129. Voir également Rickert, Die Grenzen der naturwissenschaftlichen Begriffsbildung, Tübingen, Mohr, 5e éd., 1929, p. XXIII ; Kulturwissenschaft und Naturwissenschaft, Tübingen, J.C.B. Mohr, Ge et 7e éd., 1926, p. 76; Wilhelm Windelband, p. 19.
} 
Si Simmel ne cite pas volontiers ses contemporains épistémologues, Weber en revanche, qui possède une connaissance approfondie de l'ensemble de la discussion concernant la méthodologie des sciences sociales et historiques, n’hésite pas à le faire. Ainsi dans son étude sur Roscher et Knies, il s'empresse de faire mention de la seconde édition du livre de Simmel, nouvellement parue dans une version entièrement remaniée. Or, cet article nous permet de dégager à grands traits la réaction de Weber aux avancées de la seconde édition que nous venons d'exposer. Ainsi dans une note consacrée à Simmel, Weber déclare-t-il que l'historien est clairement « lié » quant à son objet, alors qu'il est « libre » pour tout ce qui touche l'aspect « contingent» et accessoire de sa présentation. À ce dernier égard, le récit de l'historien peut sans problème avoir recours à des procédés intuitifs et à des éléments « esthétiques » ${ }^{1}$. Mais pour ce qui touche le contenu même du récit historique, il convient de quitter ce registre. En effet, l'histoire est pour Weber une discipline scientifique et comme toute science elle vise à la « connaissance », ce à quoi la conception simmelienne du discours historique finit par renoncer. Or, qui dit connaissance dit objectivité, ce qui implique une procédure de corroboration et de vérification. De cette manière, Weber se distancie de la conception de la compréhension de Simmel, qui n’hésite pas à mettre en scène l'« irrationnel » propre à l'individualité historique. Weber exige une procédure de contrôle qui permette à tous les autres chercheurs de parcourir à nouveau la démarche « logique » de l'historien, son travail de reconstruction. Nous l'avons déjà dit, c'est en dernière analyse sur l'imputation causale prise dans un sens très large que repose l'objectivité, dans la mesure où cette démarche met à contribution un savoir nomologique accessible à tous. Dans ces conditions, l'histoire n'a assurément pas à devenir elle-même une science nomothétique ${ }^{2}$. Les régularités du comportement humain ne demeurent pour elle qu'un instrument, mais un instrument indispensable pour déceler ce qui dans une individualité historique était prévisible mais aussi, le cas échéant, pour attirer l'attention sur ce qui dans l'agir humain échappe aux prévisions.

Simmel, nous l'avons vu, exploite pour sa part systématiquement le paradigme esthétique, sans craindre les conséquences que cela entraîne, notamment en regard de la conception habituelle de l'objectivité et de la vérité. D'ailleurs, on sent qu'il aurait sans doute pu faire un pas de plus dans ses développements relatifs à ce paradigme. En effet, le type d'universalité et de nécessité qui entrent en jeu en histoire rappelle étrangement les enseignements de la troisième Critique de Kant. On l'avait

\footnotetext{
${ }^{1}$ Weber, « Roscher und Knies...», p. 124; cf. « Die 'Objektivität' sozialwissenschaftlicher und sozialpolitischer Erkenntnis » (1904), p. 209; « Kritische Studien auf dem Gebiet der kulturwissenschaftlichen Logik », (1906), in ibid., p. $278-279$.

${ }^{2}$ Voir à ce sujet Wolf Feuerhahn, « Max Weber et l'explication compréhensive », Philosophie, 85, 2005, p. 33.
} 
sans doute déjà remarqué, car d'une certaine manière le rapprochement s'impose de lui-même. On songe entre autres à la « nécessité exemplaire » du jugement esthétique introduite au $\$ 18$ de cet ouvrage ainsi qu'à sa « validité exemplaire » exposée au \22. Ces deux expressions désignent en effet les modalités du jugement de goût, lequel ne repose, on le sait, sur aucun critère qui puisse mener à une démonstration en bonne et due forme. Une chose est certaine cependant, Hannah Arendt n'a quant à elle pas hésité à se réclamer de Kant à cet égard. Si c'est moins l'individualité personnelle qui l'intéresse en histoire que l'irruption de l'événement unique, de cet événement qui fait surgir la nouveauté, Arendt ressent tout de même comme Simmel le besoin d'avoir recours au modèle esthétique, explicitement kantien ici.

La plupart des concepts des sciences historiques et politiques sont de cette nature restreinte ; ils ont leur source dans un événement historique particulier et c'est ensuite que nous entreprenons de le rendre « exemplaire »- de saisir dans le particulier ce qui vaut audelà de l'unicité de l'événement ${ }^{1}$.

Arendt est dans ce texte ouvertement kantienne, mais elle est aussi, sans doute à son insu, néo-kantienne. Comme Simmel, elle sait distinguer les différentes formes d'unicité et leur valeur pour l'histoire. Comme Simmel, elle a bien saisi que l'unicité de l'événement n'intéresse l'historien que dans la mesure où il «vaut » au-delà des confins de cette unicité, qui autrement demeure synonyme de particularisme. On peut comprendre les réticences de Simmel à s'associer à l'esthétique kantienne. Dans ses leçons sur Kant publiées en 1904, ne s'était-il pas montré réservé quant à certains aspects de la troisième Critique, dont il reconnaissait néanmoins qu'elle donne le coup d'envoi à la modernité esthétique ? Pour ce qui nous intéresse tout particulièrement ici, il faut noter que Simmel est très au fait des tenants et aboutissants de la validité universelle du jugement esthétique $^{2}$. Quoi qu'il en soit, l'ironie de la situation tient à ceci que Simmel surmonte le problème de l'universalité abstraite de la loi kantienne en science naturelle et en morale par des moyens éminemment kantiens.

\footnotetext{
${ }^{1}$ Hannah Arendt, Juger. Sur la philosophie politique de Kant (1970), tr. M. Revault d'Allonnes, Paris, Le Seuil, Coll. « Points », 1991, p. 128.

2 Simmel, Kant. Sechzehn Vorlesungen, p. 211.
} 